\title{
Monolithic single mode interband cascade lasers with wide wavelength tunability
}

\author{
M. von Edlinger ${ }^{1}$, R. Weih ${ }^{2}$, J. Scheuermann ${ }^{1}$, L. Nähle ${ }^{1}$, M. Fischer ${ }^{1}$, J. Koeth ${ }^{1}$, M. Kamp ${ }^{2}$ and \\ S. Höfling ${ }^{2,3}$ \\ ${ }^{1}$ nanoplus Nanosystems and Technologies GmbH, Oberer Kirschberg 4, 97218 Gerbrunn, Germany \\ ${ }^{2}$ Technische Physik, Physikalisches Institut and Wilhelm Conrad Röntgen-Research Center for \\ Complex Material Systems, Universität Würzburg, Am Hubland, 97074 Würzburg, Germany \\ ${ }^{3}$ SUPA School of Physics and Astronomy, North Haugh, St Andrews KY16 9SS, United Kingdom
}

\begin{abstract}
Monolithic two-section interband cascade lasers offering a wide wavelength tunability in the wavelength range around $3.7 \mu \mathrm{m}$ are presented. Stable single mode emission in several wavelength channels was realized using the concept of binary superimposed gratings and two-segment Vernier-tuning. The wavelength selective elements in the two segments were based on specially designed lateral metal grating structures defined by electron beam lithography. A dual-step dry etch process provided electrical separation between the segments. Individual current control of the segments allowed wavelength channel selection as well as continuous wavelength tuning within channels. A discontinuous tuning range extending over $158 \mathrm{~nm}$ in up to six discrete wavelength channels was achieved. Mode hop free wavelength tuning up to $14 \mathrm{~nm}$ was observed within one channel. The devices can be operated in continuous wave mode up to $30^{\circ} \mathrm{C}$ with output powers of $3.5 \mathrm{~mW}$ around room temperature.
\end{abstract}

Interband cascade laser (ICL) technology has granted access to the mid-infrared wavelength region from 3 to $6 \mu \mathrm{m}$ with low threshold powers [1-4], enabling for example novel high-speed, highsensitivity tunable laser absorption spectroscopy (TLAS) applications [5,6]. These require single mode laser emission, which can be achieved by a grating structure providing distributed feedback (DFB) $[7,8]$. The spectral bandwidth of sensors based on conventional DFB lasers is however limited by the current-induced tuning range of these devices, which is usually on the order of a few nanometers. Laser sources with increased tunability offer new possibilities for spectroscopic applications, for example the simultaneous detection of several gas species with a single sensor. An increased accessible spectral range also allows base line determination in the detection of molecules with broad absorption features, e.g. complex hydrocarbons.

One way to achieve wider tunability is given by the use of a multiplexing schemes based on multiple individual DFB lasers at selected wavelengths [9]. However, this approach is expensive and limited by rapidly increasing complexity. Another approach based on external cavity (EC) setups [10] is of limited use for industrial applications due to the lack of ruggedness, the rather large size and high cost of EC based setups.

A monolithic solution that is compact, rugged and that provides high tuning speeds is given by a multi-segment laser device with aperiodic grating structures. Several competitive approaches exist like sampled gratings [11,12] and binary superimposed gratings (BSGs) [13]. In this paper BSG structures were used because of the increased coupling strength compared to alternative solutions. In addition, BSG structures allow rather readily the selection of arbitrary combinations of wavelengths within the gain area. Corresponding designs with varying numbers of segments have been demonstrated at shorter emission wavelengths based on conventional diode laser gain media [14-17]. 
In this work, two-section devices fabricated on ICL gain material emitting around $3.7 \mu \mathrm{m}$ are presented. Using individual current control of both segments, spectral mapping of the emission wavelength was performed. DFB-like emission in up to six discrete wavelength channels covering discontinuously a total tuning range of $158 \mathrm{~nm}$ is demonstrated.

The underlying operation principle of the widely-tunable lasers is based on binary superimposed gratings and a two-segment device approach for wavelength selection. A conventional DFB grating selects a single wavelength according to the Bragg condition due to the constant period of the grating structure. All other wavelengths are suppressed. A BSG is a specially designed grating structure that combines several grating periods into an aperiodic structure. This grating structure supports all imprinted Bragg wavelengths simultaneously resulting in a comb-like spectrum. A schematic example of the design process of a BSG structure is given in Figure 1 in [18]. In order to realize BSG lasers emitting light at specific wavelengths rather than emitting one or more of these BSG modes, a second BSG segment is used. By designing the BSG of this second segment to favor modes with slightly different spacing with respect to the first segment wavelength channel selection is achieved. As an example, Figure 1 depicts the simulated reflectivities of two different BSG segments designed for six wavelength channels in the wavelength range between 3.6 and $3.8 \mu \mathrm{m}$. The BSG structure was designed by transfer-matrix simulations as described in [19]. By independent current variation and therefore individual refractive index variation in both segments the BSG emission modes of the two sections can be shifted against each another. Creating different spectral overlaps that way, single mode wavelength channels can be selected by having distinct BSG modes exactly coincide for both segments. Due to lack of overlap of all other BSG modes other wavelength channels are effectively suppressed. Each selected wavelength channel can be tuned continuously by temperature or by simultaneous current variation in both segments.

The developed devices are based on six stage ICL material grown by solid source molecular beam epitaxy on GaSb substrate. The active region is surrounded by $200 \mathrm{~nm}$ thick n-doped GaSb separate confinement layers (SCL). The thicknesses of the upper and lower superlattice cladding layers were chosen to be $1970 \mathrm{~nm}$ and $3350 \mathrm{~nm}$, respectively.

In contrast to conventional DFB lasers, the widely-tunable ICL devices require the definition of a two-section device structure with suitable electrical separation between the segments. Therefore a two-step reactive ion etching (RIE) process based on a chlorine-argon-plasma was developed. In a first step, waveguide structures with two segments of equal length (defined by electron beam lithography) were etched into the upper cladding layer. Then an additional etch mask was positioned at the junction area, i.e. at the area of the trench between the two segments, and the etching was continued into the transition layer that connects the active region and the lower SCL, resulting in a final etch depth of $2.4 \mu \mathrm{m}$. A scanning electron micrograph taken at the position of the of a junction area after the second etching process is shown in Figure 2 (left). After the deposition of a thin $\mathrm{Si}_{3} \mathrm{~N}_{4}$ insulation layer, aperiodic chromium gratings with BSG patterns according to the simulations were positioned laterally beside the ridges as shown in Figure 2 (right). In accordance with the Bragg conditions for the targeted wavelengths, four to six grating periods between 525 and $550 \mathrm{~nm}$ have been superimposed in order to generate these BSGs. Subsequently, a combination of $\mathrm{Si}_{3} \mathrm{~N}_{4}$ and $\mathrm{SiO}_{2}$ passivation layers was deposited by a sputtering process on the structures. Contact windows were then opened on top of the ridges using $\mathrm{CHF}_{3}$ based RIE. The ridges were electroplated with thick $(\sim 7 \mu \mathrm{m})$ gold for improved heat removal after sputter deposition of the top contact metallization layers. Subsequently the substrate was thinned down to $150 \mu \mathrm{m}$ and provided with bottom contact layers.

After cleaving the samples into $1800 \mu \mathrm{m}$ long laser bars, these bars were coated with high reflectivity metal mirrors at the back facets and thin $\mathrm{Al}_{2} \mathrm{O}_{3}$ passivation layers at the front facets. Selected devices were then mounted epitaxial side up on TO headers with integrated thermoelectric cooler elements for characterization. 
Spectral properties of the devices were investigated using a Fourier transform infrared (FTIR) spectrometer with a spectral resolution of $0.125 \mathrm{~cm}^{-1}$ in combination with an automated setup for continuous wave $(\mathrm{CW})$ current control of the two segments. A resulting 2D mode map of a six-channel BSG device with $5.8 \mu \mathrm{m}$ wide ridges operating at $15^{\circ} \mathrm{C}$ is shown in Figure 3. Sufficient electrical separation, while avoiding the creation of individual cavities, is provided by the separation gap, that was chosen to be around $15 \mu \mathrm{m}$ long and $2 \mu \mathrm{m}$ deep, resulting in an electrical resistance around $1 \mathrm{k} \Omega$ between the segments. The gratings were designed for an effective refractive index of the optical mode around 3.5 and six emission channels around 3655, 3685, 3715, 3740, 3770 and $3810 \mathrm{~nm}$, respectively. Single mode emission wavelengths with side mode suppression ratios (SMSR) above $25 \mathrm{~dB}$ are color coded in this Figure, while black regions depict multimode emission. Four emission channels can be distinguished (marked as C, D, E, F) with central wavelengths at 3717, 3742, 3769, and $3807 \mathrm{~nm}$. These emission windows correlate with the four highest targeted wavelength channels. Within the channels DFB-like mode-hop free wavelength tuning with a current tuning coefficient of $0.09 \mathrm{~nm} / \mathrm{mA}$ was observed.

Multiple channel repetitions as well as additional single mode regions around 3750 and $3710 \mathrm{~nm}$ can be perceived. The latter might be due to emission in higher order lateral modes [20]. Since the tuning behavior of these side channels appears to be unstable, they were not considered for the determination of the tuning range of the device. Due to the spectral position of the gain with respect to the gratings only four channels could be addressed at this temperature. At an operation temperature of $5^{\circ} \mathrm{C}$, the lower three of the defined channels (A, B, C) can be addressed. Figure 4 depicts 35 selected individual spectra of this device taken at different current combinations that show an overall tuning range of $158 \mathrm{~nm}$ in six wavelength channels. The spectra are color-coded with respect to the corresponding operating temperature. One spectrum within channel $\mathrm{F}$ was depicted in black demonstrating the single mode emission. While the channels with the smallest tuning ranges are sufficient for e.g. base line sampling, up to $14 \mathrm{~nm}$ can be covered by other channels, which is comparable to the tuning range of state of the art DFB ICL devices [8,20].

Since wavelength tuning by a change in chip temperature is not fast enough for many industrial applications, a large wavelength range addressable by current tuning alone is desirable. The abovementioned device covers $95 \mathrm{~nm}$ at an operation temperature of $15^{\circ} \mathrm{C}$ (compare Figure 4). A BSG device with grating structures optimized with respect to the gain position at $15^{\circ} \mathrm{C}$ and slightly increased channel spacing shows a further enhanced maximum electrical tuning range of $120 \mathrm{~nm}$ in four channels at this temperature as presented in Figure 5.

The electro-optical properties of a fabricated BSG ICL device are presented in Figure 6. Both segments were operated with identical drive currents, representing the diagonal of a mode map. CW operation was obtained above room temperature with combined thresholds and output powers of $80 \mathrm{~mA}$ and $3.5 \mathrm{~mW}$ at $25^{\circ} \mathrm{C}$, respectively. Kinks in the P-I-curve can be attributed to wavelength channel switching. BSG laser devices exhibit 5-10\% higher thresholds compared to DFB laser devices on the same epitaxial material, mostly due to the increased losses induced by the aperiodic grating and the separation gap. Independent lasing of single segments was not observed in the presented devices.

In conclusion, two-segment laser devices with lateral BSG structures have been fabricated on GaSbbased ICL gain material emitting around $3.7 \mu \mathrm{m}$. Automated 2D measurements show defined channel switching as well as DFB-like current tuning behavior in several single mode wavelength channels, controlled by the individual drive currents in the segments. Stable wavelength tuning in up to six distinct channels with a total tuning range around $160 \mathrm{~nm}$ was achieved. An observed tuning range of up to $14 \mathrm{~nm}$ within a single channel is comparable to state of the art DFB ICL devices. The presented monolithic widely tunable devices will be suitable for a variety of applications, e.g. multi-gas-species analysis. As an outlook, this concept can be transferred to any ICL gain material to target specific wavelengths in the spectral region from 3 to $6 \mu \mathrm{m}$. In addition, 
the emission channels can be tailored greatly to exceed the continuous tuning range of DFB ICL devices in quasi-continuous tuning mode.

Financial support by the European Union in terms of the WideLase project (grant agreement no: 318798) within the Seventh Framework Programme is gratefully acknowledged.

\section{Figure Captions}

Figure 1. Simulation of the supported emission wavelengths of two BSG segments with different wavelength spacings. Each segment favors six specific wavelength channels.

Figure 2. Left: Scanning electron micrograph (SEM) of the junction area of a two-segment BSG device taken after the two-step etch process. Right: SEM of the aperiodic metal grating structures beside the ridges.

Figure 3. 2D mode map of a BSG ICL device operating at $15{ }^{\circ} \mathrm{C}$. Single mode emission wavelength is color-coded. Emission channels corresponding to the BSG structure are marked.

Figure 4. 35 individual spectra indicating the overall tuning behavior of a BSG ICL device. Six wavelength channels are observed within a spectral range of $158 \mathrm{~nm}$.

Figure 5. Tuning behavior of a BSG ICL device with $120 \mathrm{~nm}$ tuning range in four discrete channels at $15{ }^{\circ} \mathrm{C}$.

Figure 6. Electro-optical characteristics of a BSG ICL device for CW operation between 5 and $30{ }^{\circ} \mathrm{C}$.

\section{References}

[1] R. Q. Yang, Superlatt. Microstruct. 17, 77(1995).

[2] I. Vurgaftman, W. W. Bewley, C. L. Canedy, C. S. Kim, M. Kim, C. D. Merritt, J. Abell, J. R. Lindle, and J. R. Meyer, Nat. Commun. 2, 585 (2011).

[3] R. Weih, M. Kamp, and S. Höfling, Appl. Phys. Lett. 102, 231123 (2013).[4] I. Vurgaftman, R. Weih, M. Kamp, J. R. Meyer, C. L. Canedy, C. S. Kim, M. Kim, W. W. Bewley, C. D. Merritt, J. Abell, S. Höfling, J. Phys. D 48, 123001 (2015).

[5] G. Wysocky, Y. Bakhirkrin, S. So, F. K. Tittel, C. J. Hill, R. Q. Yang, and M. P. Fraser, Appl. Opt. 46, 8202 (2007).

[6] S. Lundqvist, P Kluczinsky, R. Weih, M. v. Edlinger, L. Nähle, M. Fischer, A. Bauer, S. Höfling, and J. Koeth, Appl. Opt. 51, 6009 (2012).

[7] C. S. Kim, M. Kim, W. W. Bewley, J. R. Lindle, C. L. Canedy, J. Abell, I. Vurgaftman, and J. R. Meyer, Appl. Phys. Lett. 95, 231103 (2009).[8] ～R. Weih, L. Nähle, S. Höfling, J. Koeth, and M. Kamp, Appl. Phys. Lett. 105, 071111 (2014).

[9] D. S. Baer, R. K. Hanson, M. E. Newfield, and N. K. J. M. Gopaul, Opt. Lett. 19, 1900 (1994).

[10] D. Caffey, T. Day, C. S. Kim, M. Kim, I. Vurgaftman, W. W. Bewley, J. R. Lindle, C. L. Canedy, J. Abell, and J. R. Meyer, Opt. Expr. 18, 15691 (2010).

[11] V. Jayaraman, Z. M. Chuang, and L. A. Coldren, IEEE J. Quant. Phys. 29, 1824 (1993)

[12] R. Blanchard, S. Menzel, C .Pflügl, L. Diehl, C. Wang, Y. Huang, J.-H. Ryou, R. D. Dupuls, L. Dal Negro, and F. Capasso, New J. Phys., 13, 113023 (2011).

[13] I. A. Avrutsky, D. S. Ellis, A. Tager, H. Anis, and J. M. Xu, J. Quant. Electron. 34, 729 
(1998).

[14] L. A. Coldren, Y. A. Fish, J. S. Barton, L. Johansson, and C. W. Coldren, J. Lightwave Technol. 22, 193 (2004).

[15] L. Nähle, C. Zimmermann, S. Belahsene, M. Fischer, G. Boissier, P. Grech, G. Narcy, S. Lundqvist, Y. Rouillard, J. Koeth, M. Kamp, and L. Worschech, Electron. Lett. 47, 1092 (2011).

[16] W. Zeller, M. Legge, J. Seufert, R. Werner, M. Fischer, and J. Koeth, Appl. Opt. 48, B51 (2009).[17] T. Lehnhardt, S. Höfling, M. Kamp, L. Worschech, A. Forchel, IEEE Phot. Technol. Lett. 22, 1662 (2010).

[18] M. von Edlinger, J. Scheuermann, R. Weih, L. Nähle, M. Fischer, S. Höfling, J. Koeth, and M. Kamp, Proc. SPIE 9370, 93702A (2015).

[19] M. Müller, T. Lehnhardt, K. Rößner, and A. Forchel, Appl. Phys. Lett. 93, 081117 (2008).

[20] S. Forouhar, C. Borgentun, C. Frez, R. M. Briggs, M. Bagheri, C. L. Canedy, C. S. Kim, M. Kim, W. W. Bewley, C. D. Merritt, J. Abell, I. Vurgaftman, and J. R. Meyer, Appl. Phys. Lett. 105, 051110 (2014). 


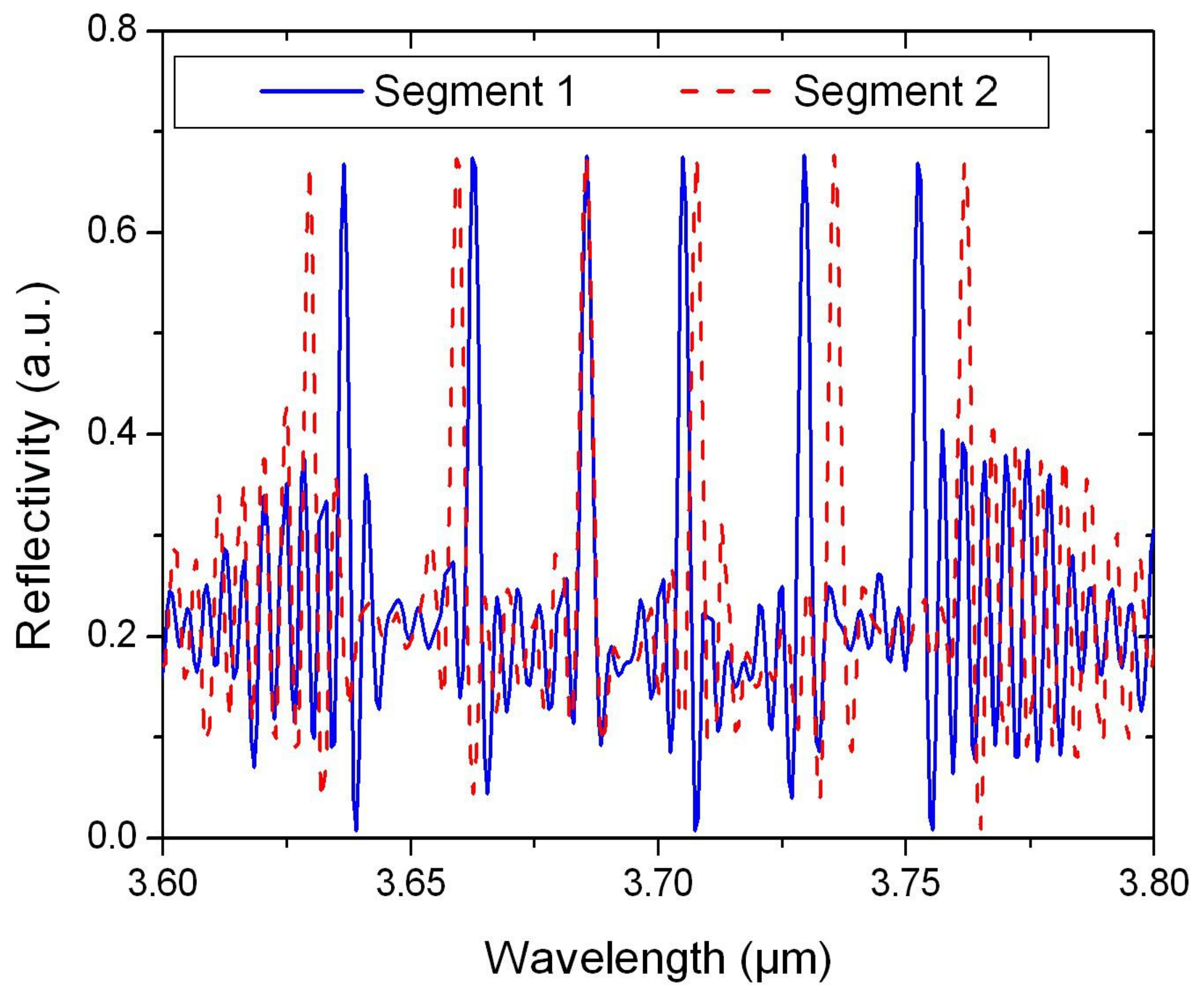




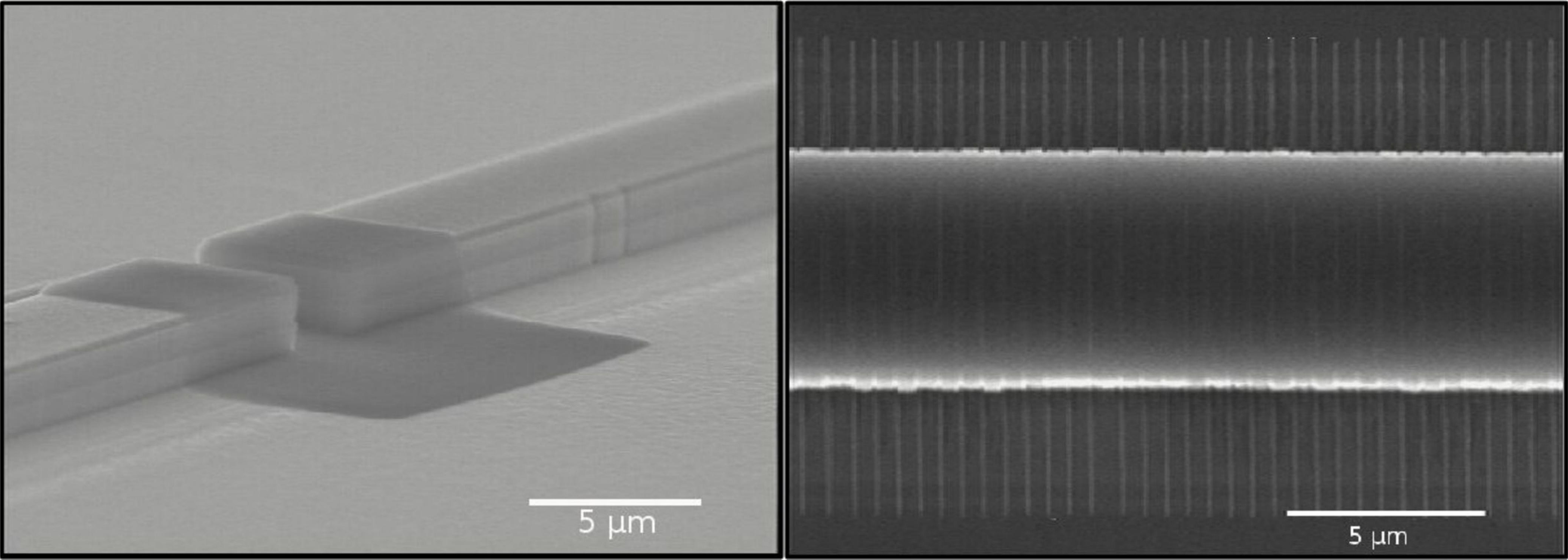




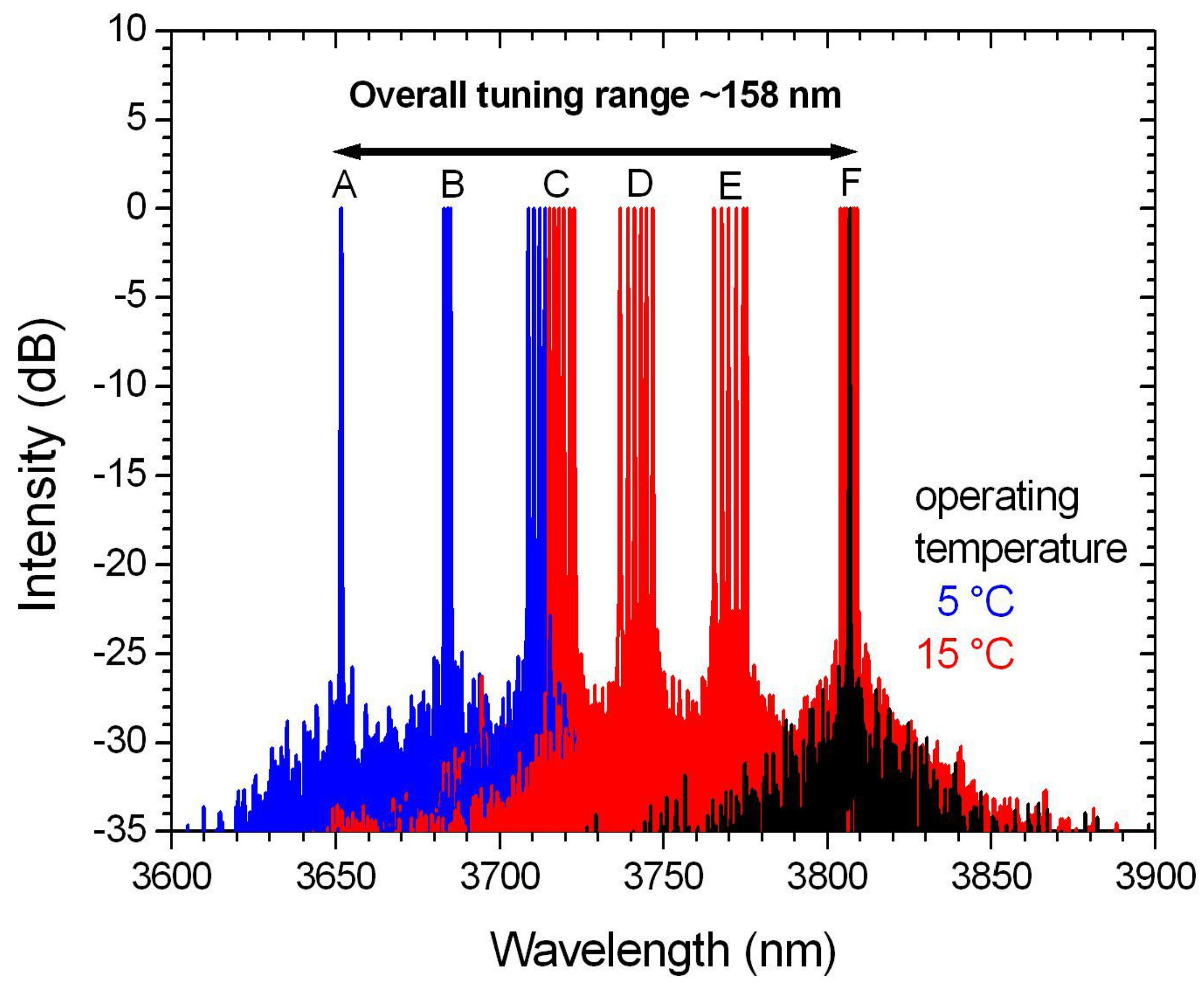




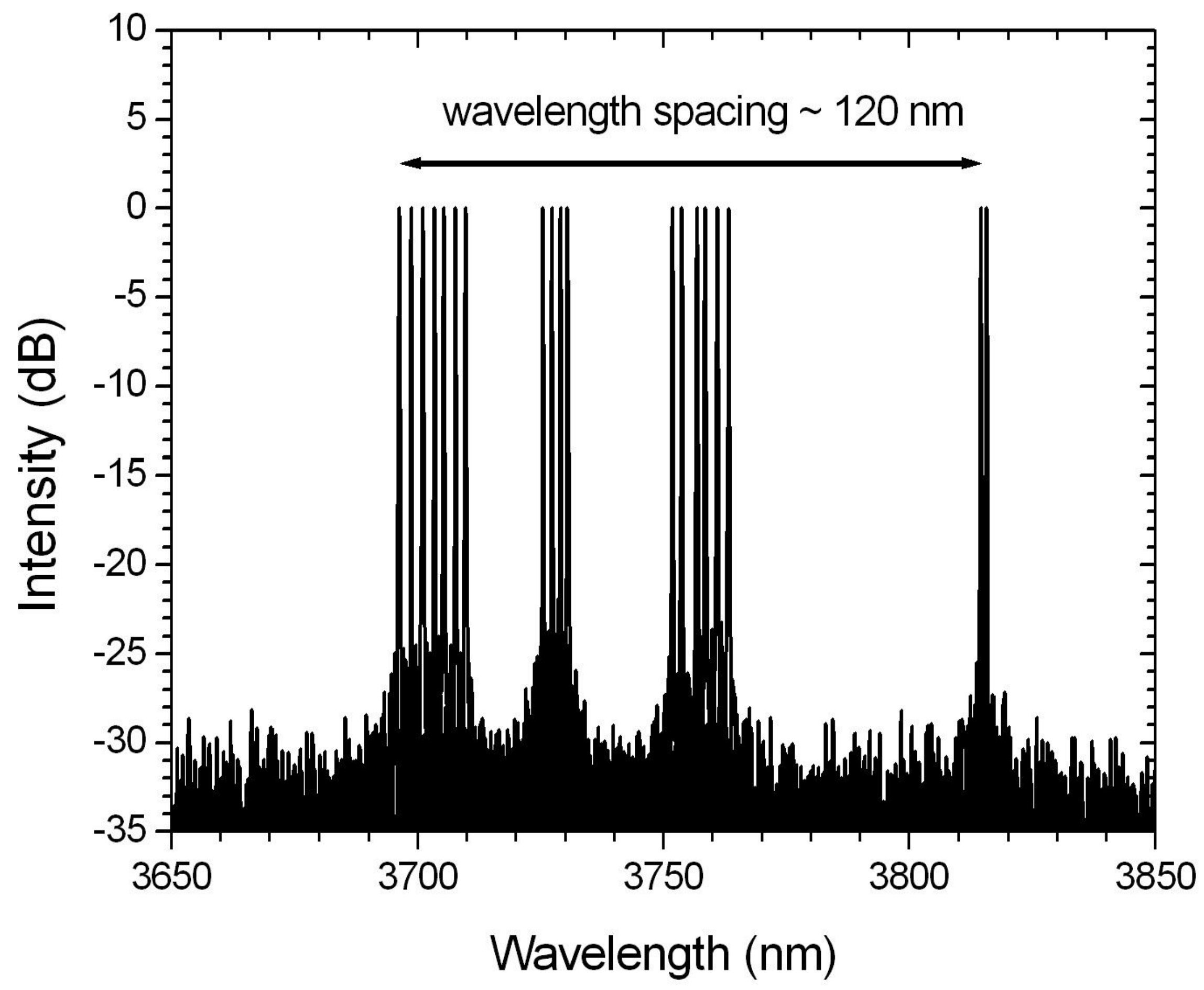




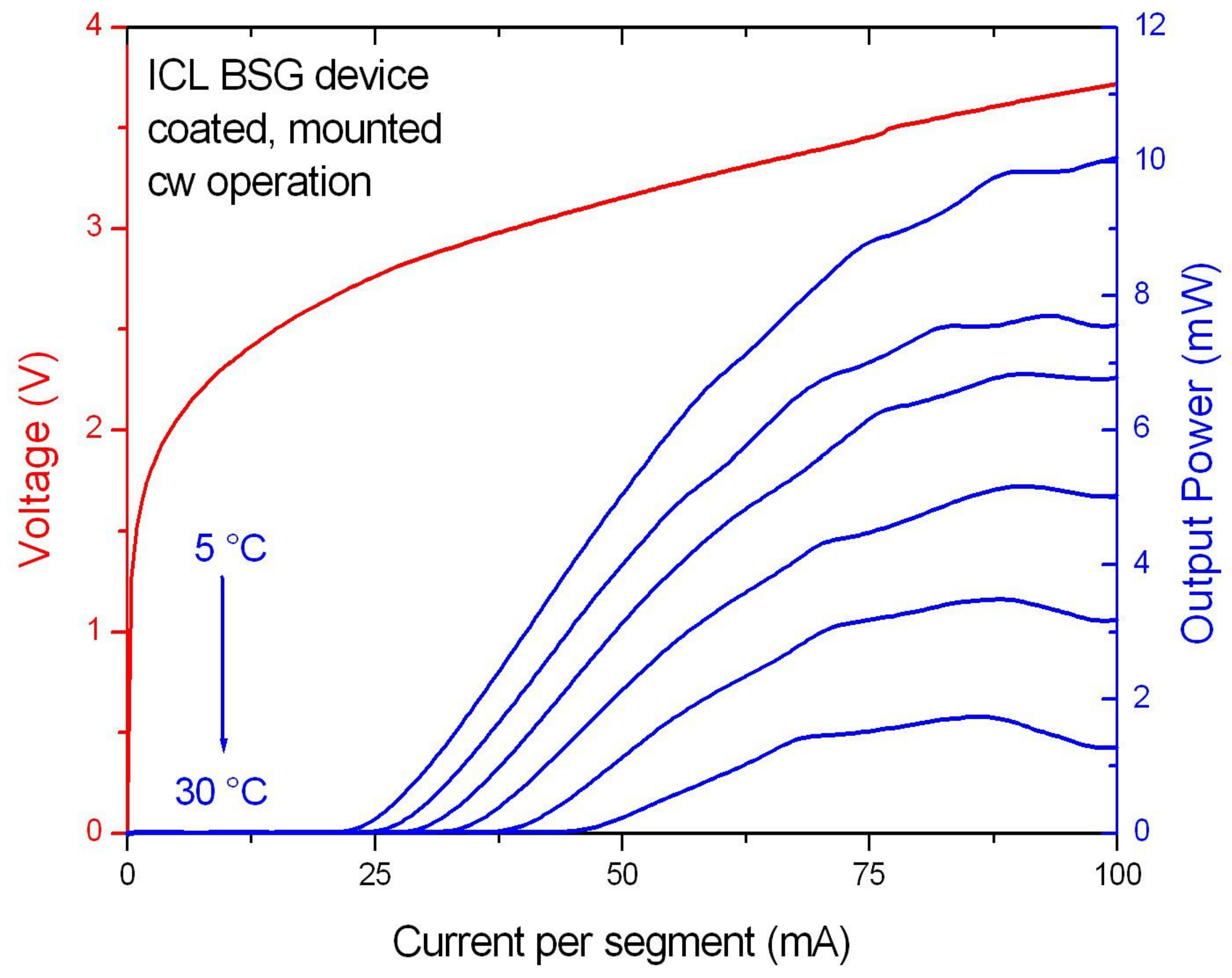

